\title{
Non-equilibrium Molecular Dynamics of Nanojet Injection in a High Pressure Environment
}

\author{
Hyun-ho Shin ${ }^{\dagger} \cdot$ Michael Oschwald ${ }^{*} \cdot$ Woong-sup Yoon $^{* *}$
}

비평형 분자동력학을 사용한 고압하의 나노제트 붕괴 수치모사

\author{
신현호 ${ }^{\dagger}$. Michael Oschwald* . 윤웅섭 ${ }^{* *}$
}

Key Words : Non-equilibrium Molecular Dynamics (비평형 분자동력학), Nanojet Injection and Breakup (나노제트 분사 및 붕괴), Critical Phenomena (임계현상)

\begin{abstract}
Using Non-equilibrium Molecular Dynamics, nanojet injection simulations have been conducted in a high pressure environment. To maintain a constant gas pressure, the periodic-shell boundary condition, which can produce a continuous gas flow with a constant temperature and velocity was used. A series of simulations were made with different chamber conditions from subcritical to supercritical pressure. Comparing the previous results from vacuum, high pressure effects accelerate jet breakup and droplet vaporization processes. Typical supercritical phenomena like the disappearance of phase interface and surface tension were also observed during the simulations.
\end{abstract}

\section{Introduction}

Most common spray combustion devices such as diesel engine and liquid rocket engine have very high pressure above the critical limit during combustion process. When the pressure is near or above the critical limit, the differences between two phases become attenuated and finally it reaches a continuous density profile without a phase interface. This 'tran/supercritical' phenomena add numerous complexities in understanding subsequent thermophysical behaviors ${ }^{(1)-(3)}$. However, the liquid jet breakup and atomization processes are well known as the controlling factor of determining the entire combustion characteristics. Therefore, the better understanding of jet breakup and atomization process under high pressure is essential for the development of higher performance spray combustion devices no matter how complicated.

As a pressure increases, both the surface tension and the heat of vaporization decrease. Especially if pressure

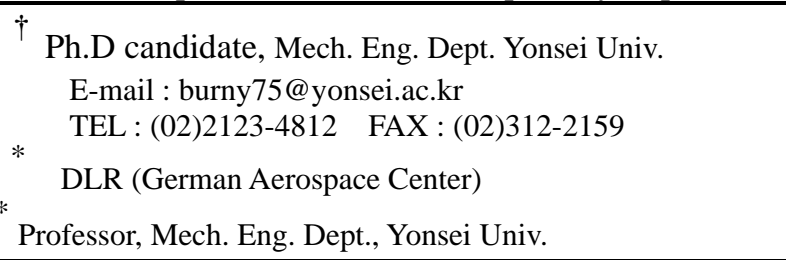

and temperature reach critical point, both surface tension and the heat of vaporization become zero. This unique behavior of critical fluid completely changes the jet breakup characteristics. However, both experimental and theoretical approaches to examine the jet breakup behaviors under high pressure have several inherent difficulties. In experimental approach, high pressure environment with large temperature and density gradient hinders optical measurements ${ }^{(1)-(3)}$. In case of a theoretic approach, phase equilibrium with the abnormal thermophysical property variation near critical point is hard to be predicted correctly. Moreover, even in stateof-art spray models, they employ many assumptions and experimental correlations which are highly casedependent ${ }^{(4)(5)}$. Generally, these inputs for spray models are not available for a high pressure environment.

As a new approach for overcoming current problems in spray modeling, Non-Equilibrium Molecular Dynamics (NEMD) have been introduced by the several researchers for the prediction of jet breakup and droplet evaporation ${ }^{(6)-(10)}$. NEMD is an ideal numerical tool for solving high pressure multi-phase problem because no additional formulas and theories are required for reproducing a high pressure effect. For example, both a phase equilibrium and properties variations are the consequences of molecular motions so that these are inherently included in NEMD. However, NEMD is a numerically-expensive method so the available 
dimensions are strictly limited up to nano meter scale. This scale problem prohibits the direct usage of NEMD into a practical scale engineering problems. Even though NEMD has a strict limit of its applicable system size, we would expect that NEMD can enhance our understanding about complex tran/supercritical jet breakup behavior. Therefore, our goal in this study is to simulate argon nanojet injection into a high pressure environment using NEMD.

\section{Applied Numerical Techniques and Simulation Setups}

This study is an expansion of our previous NEMD research about nanojet injection under vacuum condition into high pressure environment ${ }^{(11)}$. Here, the way to simulate nanojet injection will not be explained in detail because most of simulation techniques are same as the reference (11).

In this paper, all the properties are normalized. The normalization of properties were performed with the basic units in length $(\sigma)$, mass $(m)$ and energy $(\varepsilon)$. The detailed normalization procedures were introduced in the reference (11). Normalized properties are denoted by the superscript * in this paper. Applied numerical techniques in this paper are listed in table 1 . These numerical techniques are well explained in the references (11) and (12).

For the nanojet injection, a cylindrical solid platinum injector with rough surface is prepared. The shape of solid injector is in figure 1 . Liquid argon jet is continuously pushed into solid injector with a constant speed. Initial jet diameter and length are 8.81 and 470 respectively. Current jet injection method is same as previous our research so please refer to the reference (11).

Here we will mainly mention about the new features which were not used in the previous study ${ }^{(11)}$. Most significant difference in this study is the addition of gas atoms and chamber wall. Same argon is chosen as gas and a diffusive-reflection boundary condition is used to reproduce a chamber wall effect. The diffusive-reflection boundary condition showed a good agreement with some experimental results for low-density flow ${ }^{(13)}$. However, due to small chamber diameter, the jet head can act like a piston head and can accelerate gas atoms in the chamber. Therefore the no-slip wall boundary condition can be unphysical in our case. From this reason, we assumed that atoms touch the wall undergo a diffusive-reflection with a constant axial slip velocity which is equal to the jet injection velocity.

Due to the limit of available computation power, the chamber size is strictly limited. We tested various chamber diameters and chose the diameter as 35.24. Also, two different chamber lengths are used, 440.53 for low pressure cases and 367.11 for high pressure.

In spite of slip wall boundary condition, the pushing effect by the jet head is significant. This causes spatially

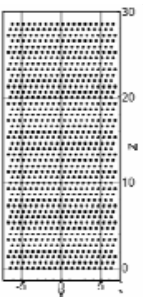

(a) Side View

$$
\text { (Y-Z Plane) }
$$

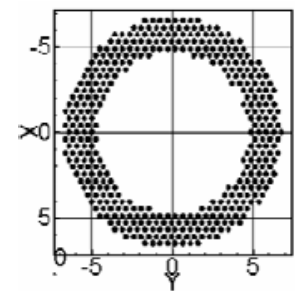

(b) Top View

(X-Y Plane)
Fig. 1 The shape of solid Pt injector. The length and inner diameter of injector are about 29.37 and 8.81 respectively.

Table. 1 Applied numerical techniques

\begin{tabular}{c|c}
\hline \hline $\begin{array}{c}\text { Interatomic } \\
\text { Potential }\end{array}$ & $\begin{array}{c}\text { Lennard-Jones 12-6 } \\
\text { Potential }\end{array}$ \\
\hline Materials & $\begin{array}{c}\text { Argon (liquid and gas), } \\
\text { Platinum (solid, FCC(111)) }\end{array}$ \\
\hline $\begin{array}{c}\text { Integration } \\
\text { Scheme }\end{array}$ & Velocity Verlet algorithm \\
\hline Timestep & $\Delta t^{*}=0.929 \times 10^{-3}$ \\
\hline $\begin{array}{c}\text { Acceleration } \\
\text { Technique }\end{array}$ & $\begin{array}{c}\text { Verlet neighbor-list method } \\
\left(r_{c}=3.0, r_{l}=3.7\right)\end{array}$ \\
\hline $\begin{array}{c}\text { Parallel } \\
\text { Computation } \\
\text { Method }\end{array}$ & Force-Decomposition \\
method (with 21 processors)
\end{tabular}

non-uniform gas density in the chamber region. To solve this problem, the easiest way is to increase the chamber diameter but this also greatly increases the computation time. Thus we decided to generate the coaxial gas flow at the inlet of chamber. The continuous gas flow with constant density and temperature was produced by the periodic-shell boundary condition ${ }^{(14)}$. Initial distribution of atoms with applied boundary conditions is shown in figure 2. Each phase is denoted by its color. Red, blue and green mean gas, liquid and solid state respectively.

As the gas density increases, an observation difficulty arises due to many gas atoms around jet surface. To get a better observation, we cut a thin slice with a thickness $3 \sigma$ along the center line. To distinguish phases clearly, we counted the number of neighbor atoms of each atom. Any atom whose distance from the opponent atom is less than $3 \sigma$ is regarded as a neighbor atom of the opponent atom in current study. The number of neighbor atoms can be used for a useful measure to define a phase ${ }^{(15)}$. This procedure is shown in figure 3 . In the following section of this paper, we have presented many figures with the contour of number of neighbor atoms. The contour color in figure 3 shows the level of neighbors of each atom. For example, the red color means high density region about 100 of neighbors. Oppositely the dark blue color in figure 3 corresponds to low density region. 
Table. 2 Simulation conditions

\begin{tabular}{|c|c|c|c|c|c|}
\hline \hline Case & $P_{r}$ & $T_{r}$ & $V_{\text {jet }}^{*}$ & $V_{\text {gas }}^{*}$ & Gas State \\
\hline 1 & - & - & 1.52 & - & Vacuum \\
\hline 2 & 0.10 & 1.19 & 1.52 & 1.52 & Subcritical \\
\hline 3 & 0.20 & 1.19 & 1.52 & 1.52 & Subcritical \\
\hline 4 & 0.41 & 1.19 & 1.52 & 1.52 & Near critical \\
\hline 5 & 0.61 & 1.19 & 1.52 & 1.52 & Near critical \\
\hline 6 & 1.22 & 1.66 & 1.52 & 1.52 & Supercritical \\
\hline
\end{tabular}

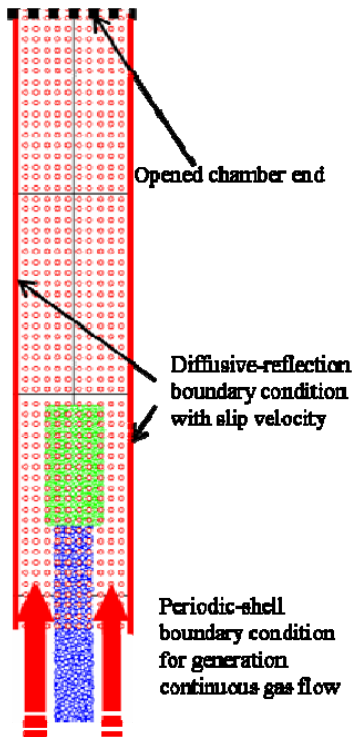

Fig. 2 Initial distribution and applied boundary conditions

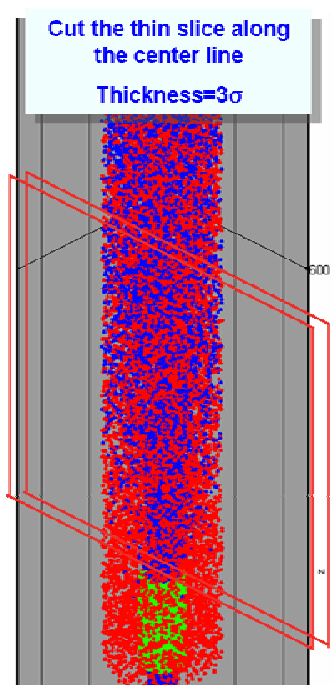

(a)

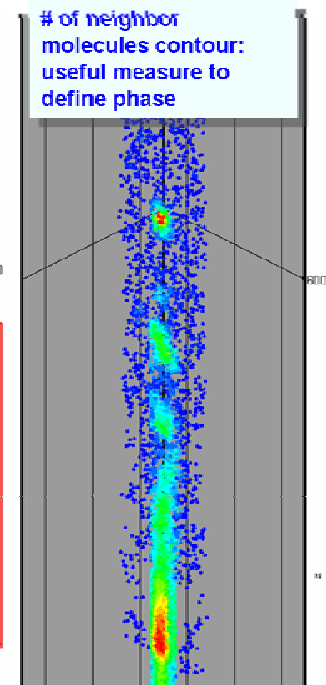

(b)
Fig. 2 Generating a slice view with the contour of neighbor atoms. (a) general 3D scatter plot in a high pressure (b) a slice view with the number of neighbors contour

\section{Results}

We made a serious of simulations with different gas pressure and velocities. The critical pressure and temperature of argon are $4.89 \mathrm{MPa}$ and $150.86 \mathrm{~K}$.

Current study focuses on the critical behavior of nanojet injection so that we will use the reduced gas pressure $\left(P_{r}\right)$ and temperature $\left(T_{r}\right)$ in the subsequent sections of this paper. Details of the simulated conditions are shown in table 2 .

\subsection{Vacuum and low pressure chamber conditions}

Before explaining the results, the understanding of high pressure phenomena is necessary. In figure 4, we show the variation of the number of neighbors and surface tension along the saturation curve of argon with the incremental saturation pressure increase. Especially the surface tension decreases rapidly and finally it becomes zero at critical pressure. However, you should keep in mind that these are valid only in bulk planar argon, not in argon nanodroplets and nanojets.

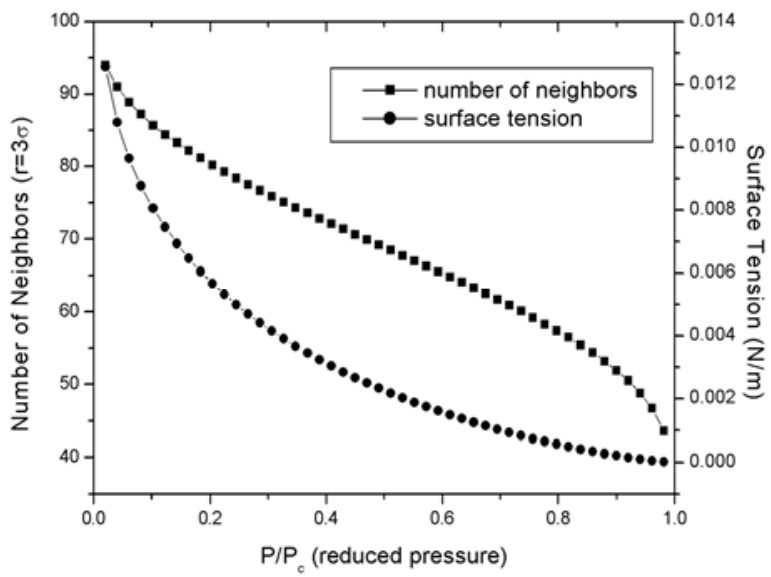

Fig. 3 Number of neighbors and surface tension in terms of reduced pressure. Number of neighbors is calculated from the bulk liquid argon density at equilibrium. Density and surface tension in this plot are obtained along bulk argon saturation curve.

First we like to compare the low pressure results (case 1, 2 and 3). Transient nanojet breakup processes are shown in figure 5 (case 1), figure 6 (case2) and figure 7 (case 3 ). In these figures, the colors of atoms show the level of neighbor atoms. In the figure 5, the first and second droplets become smaller as they move downstream. But this is because these droplets escape the $3 \mathrm{D}$ slice region along the center line. In a normal 3D plot, these droplets remain same sizes until the end of simulation. Actually there are many gas atoms but these atoms are filtered out because the number of neighbors is too low. At first glance, nanojet breakup processes in these figures look similar. The jet breakup length and droplet sizes are also comparable. 


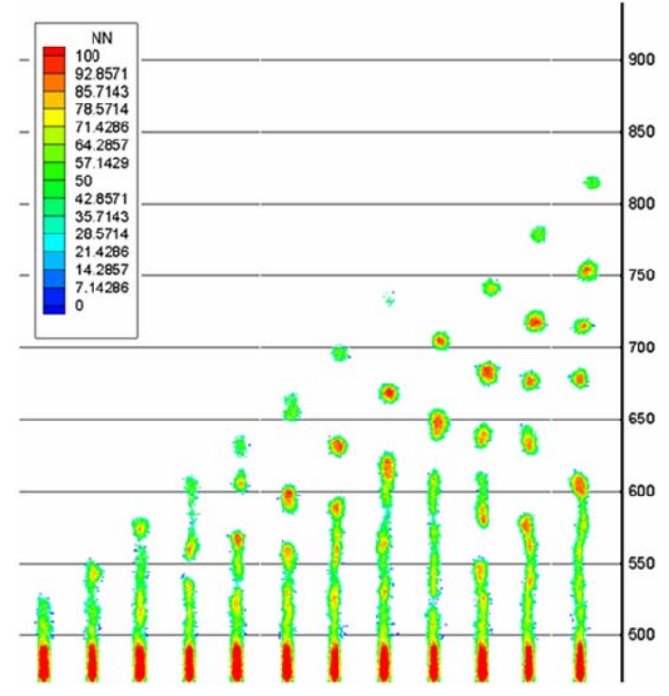

Fig. 4 Transient nanojet breakup process under vacuum condition (case 1). First plot is made at $t^{*}=46.42$ after the onset of simulation. Plotting interval is 23.21. We keep using this interval in other plots.

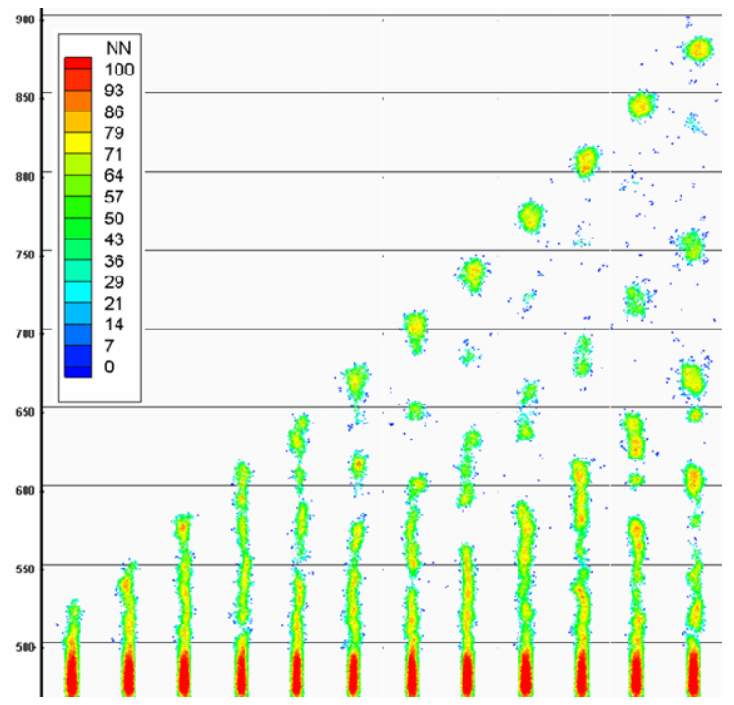

Fig. 5 Transient nanojet breakup profiles with the number of neighbor atoms contour (case 2).

However, the prominent difference of these two figures is the number of neighbors of atom in the droplets. Even small increase of gas pressure causes a significant decrease of neighbors of the atoms in the droplets. In the figure 5, the inner regions of droplets are denoted by red color which is equal to 100 neighbors approximately. On the contrary, those in figure 6 and 7 have a bright yellow color in the center of droplets. This means a significant decrease of number of neighbors. The number of neighbors can be a measure of density and potential energy. The atom with more neighbors has higher density with lower potential energy. Thus the atom with low level of potential energy needs more energy to be free (vaporization). Therefore, the increase of neighbors causes the increase of density and the heat of vaporization and the decrease of potential energy. This difference in the contour color becomes more prominent with the increase of gas pressure. The droplets in the figure 6 and 7 have less number of neighbors. Atoms with less number neighbors can be easily vaporized. Thus the subsequent droplet vaporization is faster than that from vacuum condition.

In a curved surface, the surface tension acts like a pressure. This pressure due to a curvature is called the Laplace pressure ${ }^{(16)}$. The pressure difference through a droplet interface is

$$
\Delta p=\frac{2 \gamma}{R}
$$

Here, $R$ is the radius of droplet and $\gamma$ is surface tension. In nanodroplets, $R$ is too small so that the Laplace pressure can not be negligible. Additionally the vapor pressure at the surface of small droplet can be higher than that from bulk liquid. Increased vapor pressure leads to fast droplet evaporation. The Kelvin equation speculates this behavior ${ }^{(16)}$. Thompson et al. have presented an intensive study of the size effect of nanodroplets ${ }^{(17)}$. They found that, if the droplet diameter is lower than the critical size, the deviations from the Laplace and Kelvin equations become significant. However, the droplet diameter in current study generally ranges from 10 to 15 . In this range of droplet size, the deviation from the Laplace and the Kelvin equation is marginal (17). Therefore, even though several approximations used for the derivations of these equations can be controversial in a nanodroplet, we assume the application of these equations into current study is valid.

The strong compression effect due to Laplace pressure in the nanodroplet can explain the higher density of nanodroplet than the density from bulk liquid under vacuum condition (figure 5). However, the decrease of surface tension with the pressure increase leads to the sharp decrease of Laplace pressure. Therefore, the droplets in figure 6 and 7 have lower density than the droplets under vacuum. Also, the weak density gradient at the droplet surface can be the evidence of surface tension decrease.

\subsection{Near critical and supercritical pressure chamber conditions}

We continued nanojet injection simulations with near critical (case 4, 5) and supercritical (case 6) conditions. These results are shown in figure 8, 9 and 10. Due to the decrease of surface tension, droplets in figure 8 and 9 have non-spherical shapes with obscure phase interfaces. The number of neighbors is much less than that from low pressure results. In near critical condition, droplets have too low level of cohesion force to maintain stable droplet surface. Therefore, these droplets are vaporized very quickly. In a supercritical condition (figure 10), no 


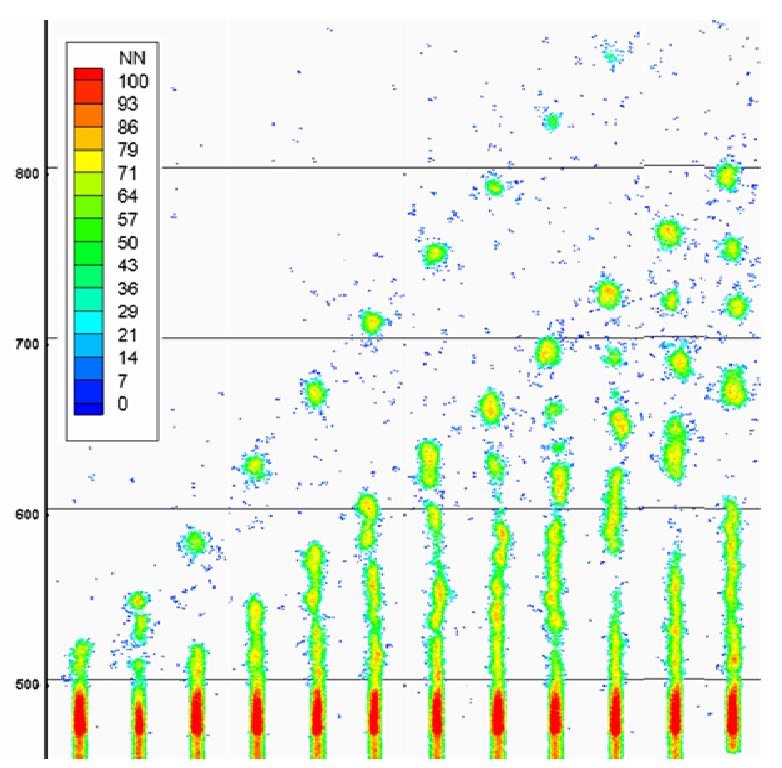

Fig. 6 Transient nanojet breakup profiles with the number of neighbor atoms contour (case 3).

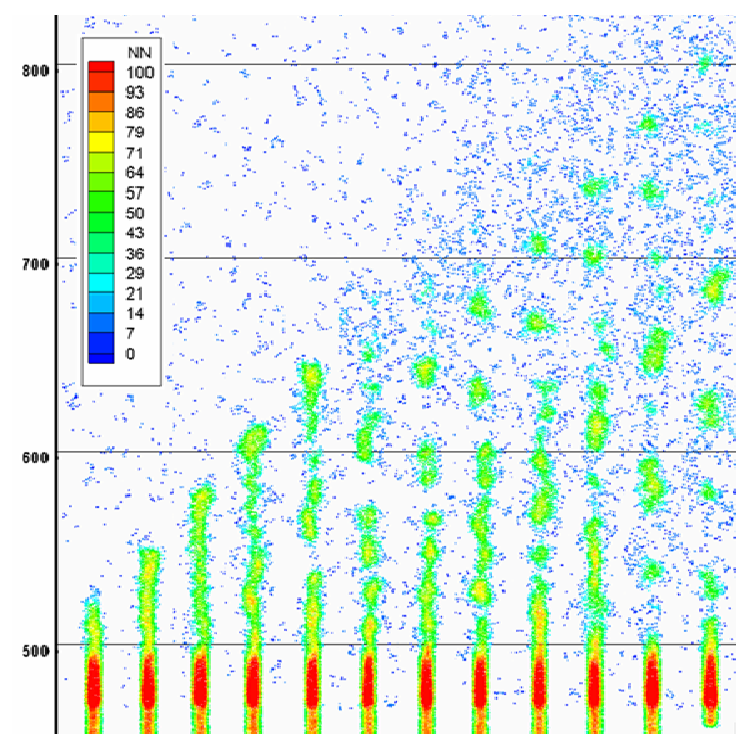

Fig. 7 Transient nanojet breakup profiles (case 4)

droplet is observed and continuous phase transition prevails. This is entirely due to the absence of both surface tension and heat of vaporization under supercritical condition. The injected nanojet in the figure 10 shows the continuous color transition from green to sky blue color.

Previous figures in lower pressure region showed the non-continuous color change from green to dark blue, which means the presence of phase interface with noncontinuous density variation. Thus, the presence of sky blue color proves that there is only one single supercritical phase with the continuous density variation.

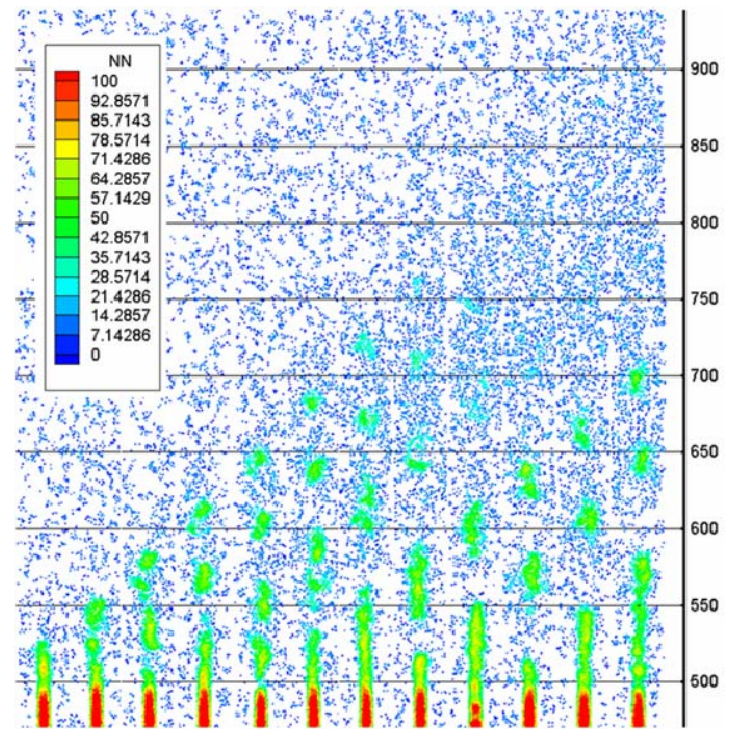

Fig. 8 Transient nanojet breakup profiles (case 5)

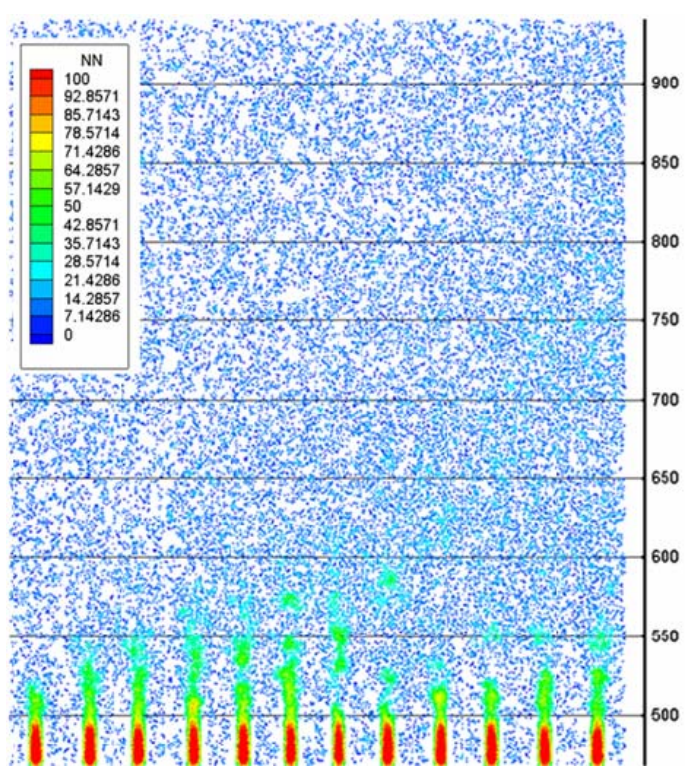

Fig. 9 Transient nanojet breakup profiles (case 6)

\section{Conclusion}

With NEMD, a series of nanojet injection simulations have been conducted from vacuum to supercritical conditions. Due to observation difficulty under high pressure, we have introduced a 3D slice view plotting technique with the number of neighbors contour. This plotting technique gives many advantages to understand complex nanojet breakup behaviors in a high pressure condition.

The droplets under vacuum have the elevated level of density due to the compression by the Laplace pressure. These droplets are very stable so that the sizes of 
droplets are almost unchanged until the end of simulations. However, even with a small pressure increases, the number of neighbors of the atoms in the droplets shows a remarkable decrease. Due to less number of neighbors, density, surface tension and the heat of vaporization are spontaneously reduced. This behavior with the elevated pressure finally causes fast droplet vaporization.

When the gas condition exceeds the critical limit, the phase interface is disappeared and the continuous phase transition dominates. These are mainly due to the absence of surface tension and heat of vaporization. Therefore, the formation of droplets is not observed under supercritical condition.

Current study can reproduce typical critical phenomena such as the disappearance of surface tension and heat of vaporization in spite of nanoscale jet diameter. Finally, it would be our next research target to increase the jet diameter with different coaxial gas flow velocities.

\section{References}

(1) Wolfgang O. H. Mayer, Axel H. A. Schik, Bruno Vielle, Christian Chauveau, Iskender Gökalp, Douglas G. Talley, Rodger D. Woodward, 1998, Atomization and Breakup of Cryogenic Propellants Under HighPressure Subcritical and Supercritical Conditions, J: Propulsion and Power, vol. 14, No. 5, pp.835-842

(2) W. Mayer, J. Telaar, R. Branam, G. Schneider, J. Hussong, 2001, Characterization of Cryogenic Injection at Supercritical Pressure, AIAA 2001-3275

(3) W. Mayer, A. Schik, B. Vielle, C. Chauveau, I. Gökalb, R. Woodward, D. Talley, T. Kaltz, L. Long, M. Micci, 1997, Experimental Investigation of Sub- and Supercritical Atomization and Gasfication, Third International Symposium on Space Propulsion Beijing, China, Aug. 11-13

(4) Yong Yi, Rolf D. Reitz, 2002, A Numerical Model for Jet Primary Breakup, ILASS Americas, $15^{\text {th }}$ Annual Conference on Liquid Atomization and Spray Systems, Madison, WI, May 2002

(5) B. S. Cheong, T. Howes, 2004, Capillary jet instability under the influence of gravity, Chem. Eng. Sci. 59, pp 2145-2157

(6) L. N. Long, M. M. Micci, B. C. Wong, 1994, Molecular Dynamics Simulations of Droplet Evaporization, AIAA 94-2907

(7) T. L. Kaltz, L. N. Long, M. M. Micci, J. K. Little, 1998, Supercritical Vaporization of Liquid Oxygen Droplets Using Molecular Dynamics, Combust. Sci. And Tech., vol. 136 pp. 279-301

(8) Micheal Moseler, Uzi Landman, 2000, Formation, Stability and Breakup of Nanojets, Science Vol. 289

(9) M. M. Micci, S. K. Oechsle, W. O. H. Mayer, 2000,
Molecular Dynamics Simulations of Primary Atomization, Eighth International Conference on Liquid Atomization and Spray Systems, Pasadena, CA. USA July 2000

(10) Kurt F. Ludwig, 2004, Molecular Dynamics Simulations of Sub- and Supercritical Injection, Master thesis Pennsylvania State University

(11) Hyun-ho Shin, Woong-sup Yoon, Michael Oschwald, 2005, Non-equilibrium Molecular Dynamics Simulation of Nanojet Breakup, KSME Spring Conference Pusan BEXCO 2005, 25-27 ${ }^{\text {th }}$ May

(12) M. P. Allen, D. J. Tildesley, 1987, Computer Simulation of Liquids, Oxford University Press

(13) J. E. A. John, 1984, Gas Dynamics, Allyn and Bacon, Inc., $2^{\text {nd }}$ Edition, Boston

(14) Akira Satoh, 1997, Periodic-shell Boundary Condition for Soft Molecular Systems: Molecular Systems: Molecular Dynamics Simulations of Flow Past a Circular Cylinder, Molecular Physics, vol. 92, No. 4, pp. 715-721

(15) Shigeo Maruyama, Sohei Matsumoto, Akihiro Ogita, 1994, Surface Phenomena of Molecular Clusters by Molecular Dynamics Method, Thermal Science \& Engineering, vol. 2, No. 1

(16) Sydney Ross, Ian D. Morrison, 1988, Colloidal Systems and Interface, John Wiley and Sons Inc.

(17) S. M. Thompson, K. E. Gubbins, J. P. R. B. Walton, R. A. R. Chanty, J. S. Rowlinson, 1984, A Molecular Dynamics Study of Liquid Drops, J. Chm. Phys. Vol. 81, No. 1 